\title{
Untying the Knot: Treating Recurrent Sigmoid Volvulus With Intravenous Levothyroxine
}

\author{
Arman Mushtaq ${ }^{\text {a, b }}$, Hiyam Bakhit ${ }^{\mathrm{a}}$, Shuvendu Sen ${ }^{\mathrm{a}}$
}

\begin{abstract}
Effect of thyroid hormone dysfunction on gastrointestinal motility is well established. Apparat from common presentation of diarrhea and constipation in hyperthyroidism and hypothyroidism respectively, some rare presentations are serious and can lead to significant morbidity and even mortality. Sigmoid volvulus is one of them, presenting as a consequence of severe longstanding hypothyroidism. We present a case of relatively young male presenting with sigmoid volvulus and treated with intravenous levothyroxine that resulted in untwisting of the volvulus without surgical intervention and endoscopic de-torsion.
\end{abstract}

Keywords: Hypothyroidism; Volvulus; IV levothyroxine

\section{Introduction}

Hypothyroidism is a common entity and gastrointestinal motility disorders are part of the horizon of signs and symptoms of this disease. Volvulus is a form of malrotation involving a loop of the bowel twisting at a focal point along the mesentery attached to the intestinal tract. Recurrent sigmoid volvulus is a rare presentation of severe hypothyroidism. Careful history and clinical judgment can prevent possible surgical intervention, if underlying hypothyroidism is recognized and promptly treated.

\section{Case Report}

A 61-year-old male presented to ER for progressive abdominal distension, colicky pain, vomiting, and lethargy for 3 days associated with 1 week of constipation followed by obsti-

Manuscript accepted for publication January 18, 2017

aDepartment of Internal Medicine, Raritan Bay Medical Center, 530 New Brunswick Ave., Perth Amboy, NJ, USA

bCorresponding Author: Arman Mushtaq, 16 Ginda Ave., Carteret, NJ 07008, USA. Email: armanmushtaq@hotmail.com

doi: https://doi.org/10.14740/jmc2750e pation for last 3 days. He had two similar episodes 2 years ago that resolved after conservative treatment with no surgical intervention. On examination, vitals were RR $18 / \mathrm{min}$, HR 80 beats/min, BP 115/66 mm Hg, temperature $97.8{ }^{\circ} \mathrm{F}$, with oxygen saturation of $98 \%$ on room air. Cardinal signs of longstanding hypothyroidism were evident as coarse facies, parotid enlargement, temporal wasting of eyebrows, husky voice, and thick dry skin. There was diffuse abdominal distension and tenderness with tympanic note and hypoactive bowel sounds. Left reducible hernia was also noted. Significant lab values were sodium 129, HB 17.9 and WBC 13. Amylase, lipase and liver function tests were within normal range. Thyroid function tests were ordered. CT urogram demonstrated sigmoid volvulus. Patient was admitted on medical/surgical floor and treated conservatively with NPO, NG tube to suction, normal saline and intravenous (IV) antibiotics. Surgical consult was requested. TSH level came back to 54 and free T4 was 0.1 . He was started on IV levothyroxine. After the initiation of IV levothyroxine, abdominal distention improved and he started to pass flatus and next day after second dose, he had a bowel movement and pain also subsided. He was started on gradually advanced diet and received total of three doses during the stay and was discharged home on oral levothyroxine with follow-up at clinic.

\section{Discussion}

Sigmoid volvulus is an uncommon cause of intestinal obstruction in United States with less than $10-15 \%$ of cases; however, in other parts of the world, it is the third most common etiology of intestinal obstructions [1]. Hypothyroidism leading to sigmoid volvulus is exceedingly rare. Although treatment of volvulus remains endoscopic de-torsion, recurrence is still common and precise pathogenesis also remains unclear. Myxedematous changes in intestinal mucosa have been postulated. Lymphocytic infiltration and hydrophilic glycoproteins deposition lead to decreased peristalsis, secondary to mechanical disruption of impulse transmission at myoneural junctions [2]. Trans-epithelial flux is affected by inhibition of $\mathrm{Cl}^{-} / \mathrm{HCO}^{3-}$ anion exchange leading to decreased peristalsis [3]. Furthermore, patients with hypothyroidism have altered anorectal physiology [4]. Most of the times, sigmoid volvulus has been reported in older and debilitated patients. In contrast, our patient was 
relatively young and not debilitated. IV levothyroxine may reverse the volvulus and avoid emergent surgery. Interestingly, use of IV levothyroxine in such circumstances has not been extensively reported and recurrent sigmoid volvulus remains among the rare presentations of severe hypothyroidism. Of note use of IV levothyroxine should be individualized according to patient's comorbidities. Cardiac and previous surgical history must be taken into account. Our patient had a recent normal echocardiogram on file and did not have any surgical history. There is sufficient evidence to support the use of IV levothyroxine as monotherapy $[5,6]$.

\section{Conclusion}

Our case highlights the importance of early recognition of underlying hypothyroidism based on history and physical examination and early administration of IV levothyroxine with low threshhold for endoscopic de-torsion and surgical intervention in carefully selected patients.

\section{References}

1. Gingold D, Murrell Z. Management of colonic volvulus. Clin Colon Rectal Surg. 2012;25(4):236-244.

2. Meytes V, Schulberg SP, Morin N, Glinik G. Undiagnosed hypothyroidism presenting with sigmoid volvulus. J Surg Case Rep. 2016;2016(4).

3. Tenore A, Fasano A, Gasparini N, Sandomenico ML, Ferrara A, Di Carlo A, Guandalini S. Thyroxine effect on intestinal $\mathrm{Cl}-/ \mathrm{HCO}_{3}{ }^{-}$exchange in hypo- and hyperthyroid rats. J Endocrinol. 1996;151(3):431-437.

4. Deen KI, Seneviratne SL, de Silva HJ. Anorectal physiology and transit in patients with disorders of thyroid metabolism. J Gastroenterol Hepatol. 1999;14(4):384-387.

5. Wu KC. Oral \#221. Presented at: American Thyroid Association Annual Meeting; Oct. 29-Nov. 2, 2014; Coronado, Calif.

6. Wiersinga WM. Paradigm shifts in thyroid hormone replacement therapies for hypothyroidism. Nat Rev Endocrinol. 2014;10(3):164-174. 